\title{
Adaptive beam forming without signal cancellation in the presence of coherent jammers
}

J.-H. Lee, BE, MSc, PhD

J.-F. Wu, BE, MSc

Indexing terms: Array processing, Antennas (arrays) (adaptive), Interference

\begin{abstract}
Adaptive beam forming using spatial smoothing has been proposed to combat coherent jammers. Recently, it has been found that this adaptive beam forming technique cannot avoid signal cancellation phenomena while also rejecting coherent jammers. In the paper, an approach is presented to eliminate the interaction between the desired signal and coherent jammers during the adaptation of spatial smoothing. As a result, the proposed beam former can effectively null coherent jammers without signal cancellation. Moreover, the resulting output signal-to-noise ratio provides the information about the existence of the desired signal. Computer simulations confirm the theoretical work.
\end{abstract}

\section{Introduction}

Research on the use of an adaptive phased-array to receive a desired signal in the look direction while nulling jammers has provided a lot of useful techniques [1]. However, these techniques are only effective in an incoherent jamming environment. Coherence between the desired signal and jammers can completely destroy the effectiveness of conventional adaptive array systems such as those proposed in References $2-7$. Coherent jammers are present in many practical situations, e.g. when multipath propagation is present or when smart jammers induce coherent interference by retrodirecting the desired signal energy to the receiving system.

To tackle the coherent jamming problem, an adaptive beam former that combines conventional adaptive array techniques with the concept of spatial smoothing has been proposed [8]. The idea of spatial smoothing was first used [9] for direction finding in coherent source situations. In the approach of Shan and Kailath [8], an array is divided into overlapping subarrays. The coherence between signals is then destroyed by adopting the spatially smoothed correlation matrix to find the optimal weight vector. Due to the fact that a coherent interferer can be viewed as a scaled and delayed version of the desired signal, the output SNR of an adaptive beam former in a coherent jamming situation is in effect $S N R=P_{s+c} / P_{n}$, where $P_{s+c}$ is the output power due to the desired signal and the coherent jammers and $P_{n}$ is the output power due to noise. Thus, the SNR should be high when the desired signal is present and low other-

Paper 6664F (E16), first received 10th March 1988 and in final revised form 8th February 1989

The authors are with the Department of Electrical Engineering, National Taiwan University, Taipei, Taiwan, Republic of China

IEE PROCEEDINGS, Vol. 136, Pt. F, No. 4, AUGUST 1989 wise. However, it has been shown by Reddy et al [11] that the spatial smoothing technique of Shan and Kailath [8] cannot cope with the coherent jamming problem without signal cancellation. Therefore, the output SNR cannot be used to indicate the existence of the desired signal. Furthermore, the performance of the array would not be satisfactory when the power level of coherent jammers is low. Hence, the signal cancellation effect due to the interaction between the desired signal and the coherent jammers in an adaptive beam former has been attracting much attention. Widrow et al. [10] have proposed an adaptive beam forming scheme using two beam formers (master and slaved beam formers) to separate the signal and the jammers during adaptation. The Frost adaptive beam former is employed to generate a suitable set of weights to satisfy the look-direction gain constraint and to minimise the output power. The weights are then deployed in the slaved beam former to provide jammer rejection without signal cancellation. Recently [12], the spatial smoothing scheme of Shan and Kailath [8] has been modified and used to replace the conventional Frost adaptive beam former in the structure of Reference 10 .

In this paper, we propose an algorithm which can create an adaptive beam former to reject coherent jammers while providing the desired signal reception in the look direction. The desired signal is removed from the received signal in an adaptive process which is additional to the adaptive procedure of the spatial smoothing scheme. After the spatial smoothing process, we obtain an appropriate set of weights and an estimate of the desired signal. A slaved beam former based on the set of weights is then utilised to achieve the goal of coherent jammer rejection while simultaneously preserving the desired signal.

\section{Formulation of the new adaptive beam former}

Consider the collinear array of $L$ equally spaced elements shown in Fig. 1. $K+1$ coherent signals are incident on it in plane waveform at different angles $\left\{\theta_{k}, k=0,1, \ldots\right.$,

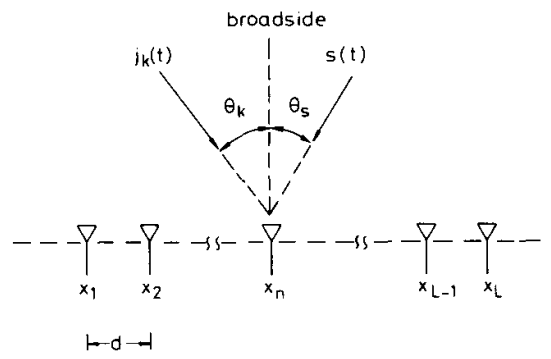

Fig. 1 Array scenario 
$K$ \}. Suppose that the desired signal $s(t)$ is of the form $s(t)=\rho_{s} \exp \left(j \omega_{0} t\right)$ and at an angle $\theta_{0}$, where $\rho_{\mathrm{s}}$ is the complex envelope of $s(t)$. The other $K$ coherent signals are assumed to be jamming signals of the form $j_{k}(t)=$ $\rho_{k} \exp \left(j \omega_{0} t\right)$, for $k=1,2, \ldots, K$, with complex envelope $\rho_{k}$. Let $\alpha_{s}=\exp \left[-j \omega_{0}\left(d \sin \theta_{s}\right) / c\right]$ and $\alpha_{k}=$ $\exp \left[-j \omega_{0}\left(d \sin \theta_{k}\right) / c\right]$ be the interelement phase shifts for $s(t)$ and $j_{k}(t)$, respectively, where $d$ is the spacing between adjacent array elements and $c$ is the propagation velocity of the plane waves. The received signal at the $l$ th array element can then be written as

$$
\begin{aligned}
x_{l}(t)= & s(t) \alpha_{s}^{l-1}+\sum_{k=1}^{K} j_{k}(t) \alpha_{k}^{l-1}+v_{l}(t) \\
= & \exp \left(j \omega_{0} t\right)\left\{\rho_{s} \alpha_{s}^{l-1}+\sum_{k=1}^{K} \rho_{k} \alpha_{k}^{l-1}\right\}+v_{l}(t), \\
& l=1, \ldots, L
\end{aligned}
$$

where $v_{t}(t)$ is the additive white Gaussian noise (AWGN) received at the lth element with $E\left\{v_{1}(t)\right\}=0$ and $E\left\{v(t) v_{m}^{*}(t)\right\}=\sigma_{v}^{2} \delta(l-m)$. We now feed the received signal of eqn 1 into the array processor shown in Fig. 2. $\eta \alpha_{s}^{i-1} d(t)$ is first subtracted from $x_{f}(t)$ to give a residual signal $z_{i}(t)$ :

$$
z_{l}(t)=x_{l}(t)-\eta \alpha_{s}^{l-1} d(t), \quad l=1,2, \ldots, L
$$

where $\eta$ is a complex number and $d(t)=\exp \left(j \omega_{0} t\right)$ is used as a reference signal. The residual signals of eqns. 2 are divided into overlapping subgroups of $M$ samples each as follows:

$$
\begin{aligned}
& h^{(1)}(t)=\left\{z_{1}(t), z_{2}(t), \ldots, z_{M}(t)\right\}^{T}=x^{(1)}(t)-\eta d^{(1)}(t), \\
& \boldsymbol{h}^{(n)}(t)=\left\{z_{n}(t), z_{n+1}(t), \ldots, z_{n+M-1}^{(n)}\right\}^{\mathrm{T}} \\
& =x^{(n)}(t)-\eta d^{(n)}(t) \\
& x^{(n)}(t)=\left\{x_{n}(t), x_{n+1}(t), \ldots, x_{n+M-1}(t)\right\}^{\mathbf{T}} \\
& \boldsymbol{d}^{(n)}(t)=\left\{\alpha_{s}^{n-1} \exp \left(j \omega_{0} t\right), \ldots, \alpha_{s}^{n+M-2} \exp \left(j \omega_{0} t\right)\right\}^{\mathrm{T}}
\end{aligned}
$$$$
\text { where }
$$

and

Next, define the steering vector of the desired signal by

$$
s_{d}=\left\{1, \alpha_{s}, \ldots, \alpha_{s}^{M-1}\right\}^{\mathrm{T}}
$$

and a spatially smoothed correlation matrix [8] by

$$
\bar{R}=\frac{1}{N} \sum_{n=1}^{N} E\left\{h^{(n)}(t) h^{(n)^{\mathrm{H}}}(t)\right\}=\frac{1}{N} \sum_{n=1}^{N} R_{n}
$$

where $N$ denotes the number of subgroups, $R_{n}$ the correlation matrix associated with the $n$th data vector $h^{(n)}(t)$, and $H$ the Hermitian transpose. Then, eqn. 5 can be

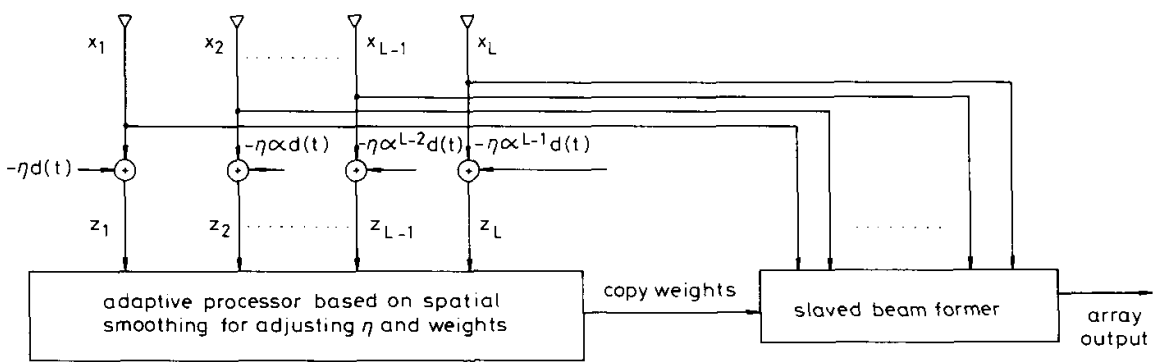

Fig. 2 Proposed adaptive beam former

170

IEE PROCEEDINGS, Vol. 136, Pt. F, No. 4, AUGUST 1989 rewritten as

$$
\bar{R}=\frac{1}{N} \sum_{n=1}^{N}\left\{R_{x x}^{(n)}-\eta^{*} \gamma_{d x}^{(n) *}-\eta \gamma_{d x}^{(n)}+|\eta|^{2} \gamma_{d d}^{(n)}\right\}
$$

where $\gamma_{d x}^{(n)}=E\left\{d^{(n)}(t) x^{(n) \mathrm{H}}(t)\right\}$ and $\gamma_{d d}^{(n)}=E\left\{d^{(n)}(t) d^{(n)^{\mathrm{H}}}(t)\right\}$. Using the criterion of the spatial smoothing scheme and letting $\boldsymbol{W}$ be the weight vector, we get the following minimisation problem:

\section{minimising $\boldsymbol{W}^{\mathrm{H}} \bar{R} \boldsymbol{W}$}

subject to $W^{\mathbf{T}} s_{d}=C=$ constant

The minimisation problem expr. 7 can be rewritten as

$$
\begin{array}{r}
\text { minimising } W^{\mathrm{H}} \bar{R} \boldsymbol{W}=\operatorname{minimising} \frac{1}{N} \sum_{n=1}^{N} W^{\mathrm{H}} R_{n}^{*} W \\
=\operatorname{minimising} \frac{1}{N} \sum_{n=1}^{N} E\left\{\left|\boldsymbol{W}^{\mathrm{T}} h^{(n)}(t)\right|^{2}\right\}
\end{array}
$$

From relation 9, we note that the minimisation problem 7 and 8 can be accomplished by minimising $E\left\{\left|W^{\mathrm{T}} h^{(n)}(t)\right|^{2}\right\}$ subject to $W^{\mathrm{T}} s_{d}=C$ for $n=1,2, \ldots, N$, i.e. by minimising the mean-squared output of each array subgroup under the constraint $W^{\mathrm{T}} s_{d}=C$ if $M$, $N \geqslant K+1$. From the definition of $h^{(n)}(t), E\left\{\left|W^{\mathrm{T}} \boldsymbol{h}^{(n)}(t)\right|^{2}\right\}$ can be written as follows

Substituting $x^{(n)}(t)$ and $d^{(n)}(t)$ into eqn. 10 yields

$$
\begin{aligned}
E & \left\{\left|\boldsymbol{W}^{\mathrm{T}} \boldsymbol{h}^{(n)}(t)\right|^{2}\right\} \\
= & E\left\{\mid \sum_{m=1}^{M} w_{m}\left[\left(\rho_{s}-\eta\right) \alpha_{s}^{m+n-2}+\sum_{k=1}^{K} \rho_{k} \alpha_{k}^{m+n-2}\right] \exp \left(j \omega_{0} t\right)\right. \\
& \left.+\left.\sum_{m=1}^{M} w_{m} v_{m+n-1}(t)\right|^{2}\right\} \\
= & \left|\left(\rho_{s}-\eta\right) \alpha_{s}^{n-1} C+\sum_{k=1}^{K} \rho_{k} \alpha_{k}^{n-1} B_{k}\right|^{2}+P_{v}^{(n)}
\end{aligned}
$$

where $B_{K}=\sum_{m=1}^{M} w_{m} \alpha_{k}^{m-1}$ denotes the beam pattern due to the weight vector $W$ at $\theta_{k}$ and $P_{v}^{(n)}$ the output power due to the noise at the $n$th subgroup. Now suppose that $P_{v}^{(n)}$ is very small or negligible. Then minimising eqn. 11 requires that the square term of eqn. 11 should approach zero. Ideally, we obtain the following set of equations:

$$
\begin{aligned}
&\left(\rho_{s}-\eta\right) C+\rho_{1} B_{1}+\rho_{2} B_{2}+\cdots+\rho_{K} B_{K}=0 \\
&\left(\rho_{s}-\eta\right) \alpha_{s} C+\rho_{1} \alpha_{1} B_{1}+\cdots+\rho_{K} \alpha_{K} B_{K}=0 \\
& \vdots \\
&\left(\rho_{s}-\eta\right) \alpha_{s}^{N-1} C+\rho_{1} \alpha_{1}^{N-1} B_{1}+\rho_{2} \alpha_{2}^{N-1} B_{2} \\
&+\cdots+\rho_{K} \alpha_{K}^{N-1} B_{K}=0
\end{aligned}
$$

$$
E\left\{\left|W^{\mathrm{T}} h^{(n)}(t)\right|^{2}\right\}=E\left\{\left|W^{\mathrm{T}} x^{(n)}(t)-\eta W^{\mathrm{T}} d^{(n)}(t)\right|^{2}\right\}
$$


If we consider the term $\rho_{s}-\eta$ as a variable $\beta$, then eqns 12 form a set of linearly homogeneous equations with variables $\beta, B_{1}, \ldots, B_{K}$. Furthermore, since $\left\{1, \alpha_{s}, \ldots\right.$. $\left.\alpha_{s}^{N-1}\right\}^{\mathrm{T}}$, and $\left\{1, \alpha_{k}, \ldots, \alpha_{k}^{N-1}\right\}^{\mathrm{T}}, k=1, \ldots, K$, are linearly independent, the solution of eqn. 12 will be $\beta=B_{1}=$ $B_{2}=\cdots=B_{K}=0$ provided that $M, N \geqslant K+1$. Therefore, the resulting array pattern has nulls in the directions of the jammers, $\theta_{1}, \theta_{2}, \ldots, \theta_{R}$ while satisfying the main beam constraint. Moreover, since $\beta=\rho_{s}-\eta=0$, i.e. $\eta=\rho_{s}$, the complex envelope of the desired signal can be estimated. This additional information is useful for determining the existence of the desired signal.

\section{Implementation of the new adaptive array}

In this Section, we consider the implementation of the proposed array described above. As shown in Fig. 2, the reference signal with unknown envelope $\eta d(t) \alpha_{s}^{l-1}$ is subtracted from the received signal $x_{f}(t)$ at the $l$ th sensor to form the difference signal $z_{l}(t)$ of eqn. 2 . The difference signals $z_{l}(t)$ are then fed into the adaptive beam former to adaptively adjust the variable $\eta$ and array weights, $w_{1}$ $w_{2}, \ldots, w_{M}$ to achieve the minimisation expressions 7 and 8.

Let us proceed with eqn. 10. From the definition of $d^{(n)}(t)$, the term $W^{\mathrm{T}} d^{(n)}(t)$ of eqn. 10 is given by

$$
W^{\mathrm{T}} d^{(n)}(t)=\alpha_{s}^{n-1} \exp \left(j \omega_{0} t\right) W^{\mathrm{T}} s_{d}
$$

Substituting the constraint eqn. 8 into eqn. 13 , we have

$$
W^{\mathrm{T}} d^{(n)}(t)=C \alpha_{s}^{n-1} \exp \left(j \omega_{0} t\right) \triangleq d_{n}(t)
$$

Consequently, eqn. 10 can be rewritten as

$$
\begin{aligned}
E\left\{\left|W^{\mathrm{T}} x^{(n)}(t)-\eta W^{\mathrm{T}} d^{(n)}(t)\right|^{2}\right\} \\
=E\left\{\left|W^{\mathrm{T}} x^{(n)}(t)-\eta d_{n}(t)\right|^{2}\right\}
\end{aligned}
$$

From eqn. 15 we obtain

$$
\begin{aligned}
& E\left\{\left|\boldsymbol{W}^{\mathrm{T}} \boldsymbol{x}^{(n)}(t)-\eta d_{n}(t)\right|^{2}\right\} \\
& =\boldsymbol{W}^{\mathrm{H}} \boldsymbol{R}_{x x}^{(n)} \boldsymbol{W}-\eta \boldsymbol{W}^{\mathrm{H}} \boldsymbol{\gamma}_{d_{n} x}^{*}-\eta^{*} \boldsymbol{W}^{\mathrm{T}} \gamma_{d_{n} x}+|\eta|^{2}|C|^{2}
\end{aligned}
$$

Therefore, the minimisation problem becomes:

$$
\begin{aligned}
& \text { minimising } e^{2}=\sum_{n=1}^{N} E\left\{\left|W^{\mathrm{T}} x^{(n)}(t)-\eta d_{n}(t)\right|^{2}\right\} \\
& \text { subject to } \quad W^{\mathrm{T}} s_{d}=C
\end{aligned}
$$

Next, we introduce the Lagrange multiplier $\lambda$ to obtain the equivalent unconstrained minimisation problem as follows:

$$
\text { minimising } \begin{aligned}
e_{1}^{2} & =\frac{1}{N} \sum_{n=1}^{N} E\left\{\left|W^{\mathrm{T}} \boldsymbol{x}^{(n)}(t)-\eta d_{n}(t)\right|^{2}\right\} \\
& -\lambda\left(\boldsymbol{W}^{\mathrm{T}} \boldsymbol{s}_{d}-C\right)^{*}-\lambda^{*}\left(\boldsymbol{W}^{\mathrm{T}} \boldsymbol{s}_{d}-C\right)
\end{aligned}
$$

Eqn. 19 indicates that the performance measure is a real quadratic function of $\boldsymbol{W}$ and $\eta$. Using the gradient method, we take the derivatives of $e_{1}^{2}$ with respect to the weight vector $\boldsymbol{W}$ and the variable $\eta$, respectively, and let $C$ equal one. The results are given as follows

$$
\nabla_{W}\left(e_{1}^{2}\right)=2\left(\bar{R}_{x} W-\eta \bar{\gamma}^{*}-\lambda s_{d}^{*}\right)
$$

and

$$
\partial e_{1}^{2} / \partial \eta=-2\left(\boldsymbol{W}^{\mathrm{T}} \bar{\gamma}-\eta / N\right)
$$

where

$$
\bar{R}_{\mathrm{x}}=\frac{1}{N} \sum_{n=1}^{N} R_{x x}^{(n)}
$$

IEE PROCEEDINGS, Vol. 136, Pt. F, No. 4, AUGUST 1989 and

$$
\bar{\gamma}=\frac{1}{N} \sum_{n=1}^{N} \gamma_{d_{n} x}
$$

Letting eqn. 21 equal zero gives the optimal value of $\eta$ in terms of the optimal weight vector $\boldsymbol{W}_{\mathrm{opt}}$

$$
\eta_{o p t}=N \bar{\gamma}^{\mathbf{T}} \boldsymbol{W}_{\text {opt }}
$$

Substituting eqn. 22 into eqn. 20 and letting $\nabla_{W}\left(e_{1}^{2}\right)$ equal zero, we obtain the optimal weight vector

$$
W_{o p t}=\lambda\left(\bar{R}_{x}-N \bar{\gamma}^{*} \bar{\gamma}^{T}\right)^{-1} s_{d}^{*}
$$

The optimal weight vector is then utilised in a slaved beam former to generate the array output. We note from eqn. 23 that the effect of subtracting $\eta \alpha_{s}^{l-1} \exp \left(j \omega_{0} t\right)$ from $x_{l}(t)$ as given by eqn. 2 is to eliminate the contribution of the desired signal component to the spatially smoothed correlation matrix by $\boldsymbol{N}^{*} \bar{\gamma}^{-\mathrm{T}}$. Furthermore, eqn. 23 reveals that the desired signal can be excluded iteratively during the minimisation procedure. Therefore, the resulting array processor tends to suppress all signals involved in the correlation matrix $\bar{R}_{x}$ besides the signal coming from the direction constrained by the steering vector $s_{d}$. Consequently, the array pattern due to the optimal weight vector will provide a peak in the constraint direction no matter whether the desired signal exists or not.

In the following, we consider the adaptive algorithm for the proposed beam former. Since the associated performance measure of the minimisation problem in eqn. 19 is in quadratic form, therefore any gradient-based algorithm can be employed to iteratively adjust the weight settings and the variable $\eta$. For instance, the iterative equations using the gradient-based method are given as follows:

$$
\begin{aligned}
& w_{i}(k+1)=w_{i}(k)-\hat{a} \frac{\partial e_{1}^{2}}{\partial w_{i}}, i=1,2, \ldots, M \\
& \eta(k+1)=\eta(k)-\hat{a} \frac{\partial e_{1}^{2}}{\partial \eta}
\end{aligned}
$$

with initial values $w_{i}(0)=\eta(0)=0, i=1,2, \ldots, M$, where $\hat{a}$ is the step size parameter. The gradients $\partial e_{1}^{2} / \partial w_{i}$ and $\partial e_{1}^{2} / \partial \eta$ are given in eqns. 20 and 21 , respectively. Alternatively, the direct method may be considered. In this case, what we have to compute at the $k$ th iteration is $\left\{\bar{R}_{x}\right.$ $\left.-N \bar{\gamma}^{*} \bar{\gamma}^{\mathrm{T}}\right\}^{-1}$. A simple recursive equation, such as the RLS algorithm utilising matrix inversion formulae can be used to estimate the value of $\left\{\bar{R}_{x}(k)-N \bar{\gamma}^{*}(k) \bar{\gamma}^{-\mathrm{T}}(k)\right\}^{-1}$

\section{Computer simulations}

Several simulation examples are presented in this Section to demonstrate the performance of the proposed adaptive beam-forming algorithm. These simulations were performed under the following coherent source environment. The desired signal with complex envelope $\rho_{s}=6+j 8$ was at an angle $\theta_{s}=0^{\circ}$. Two coherent jammers with $\rho_{1}=(10)^{1 / 2}$ and $\rho_{2}=(-0.8+j 0.6)(10)^{1 / 2}$ arrived at angles $\theta_{1}$ and $\theta_{2}$ such that $\sin \theta_{1}=-0.29$ and $\sin \theta_{2}=$ 0.29 , respectively. The signal-to-each-jammer power ratio was thus $10 \mathrm{~dB}$ and the input signal-to-noise ratio (SNR) was set equal to $20 \mathrm{~dB}$. A ten-element linear array was partitioned into four subgroups with a size of seven elements. For all simulations, 800 snapshots were used to estimate the correlation matrices $R_{n}$ of eqn. 5 . 
Fig. 3 shows the resulting array beam patterns using the conventional spatial-smoothing algorithm [8].
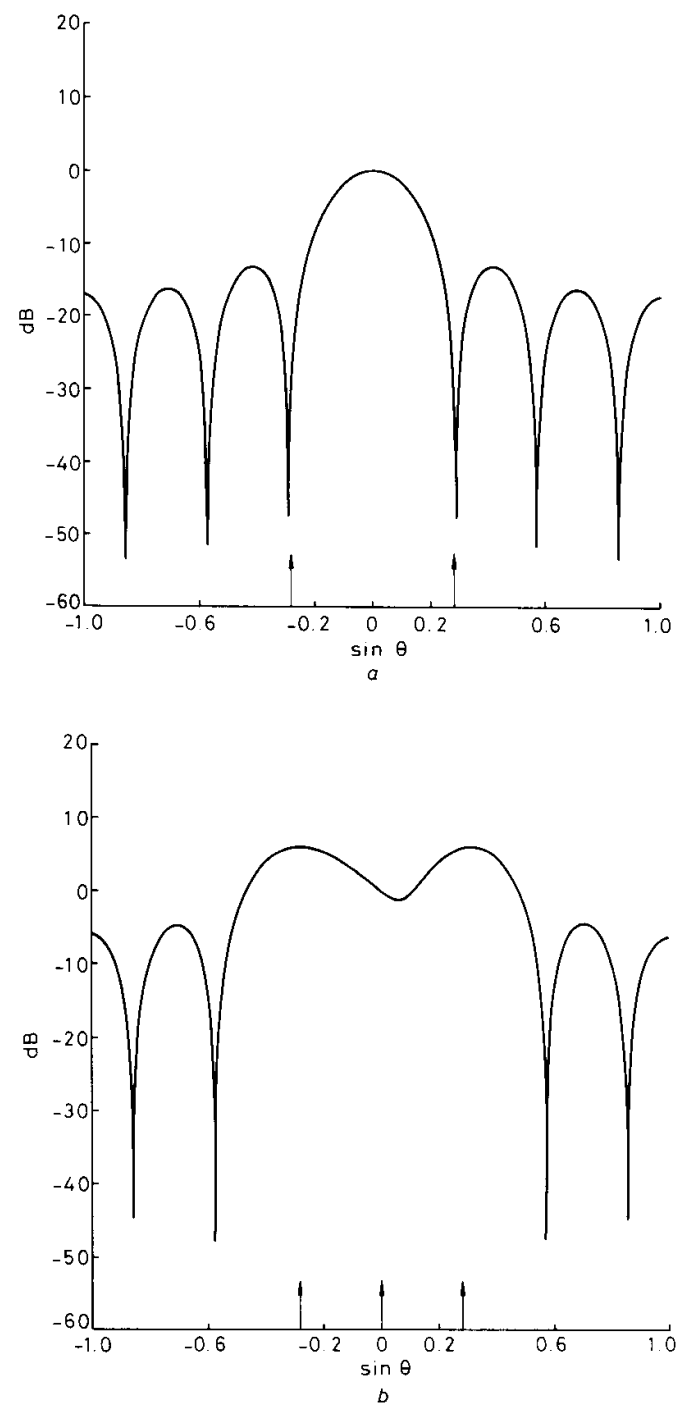

Fig. 3 Array patterns using spatial smoothing method $a$ Desired signal absent $b$ Desired signal present

Although the array pattern is acceptable when the desired signal is absent, the signal cancellation effect is very severe when the desired signal is present. Moreover, the output SNR is about $-122 \mathrm{~dB}$ when there is no desired signal, whereas it is about $21 \mathrm{~dB}$ when the desired signal is present. In contrast, the array beam patterns using the proposed adaptive beam former are shown in Fig. 4. These two array patterns are almost identical, regardless of the existence of the desired signal. The corresponding output SNR and $\eta_{\text {opt }}$ are about $-113 \mathrm{~dB}$ and zero, respectively, when the desired signal is absent. They are about $31 \mathrm{~dB}$ and $6+j 8$, respectively, if the desired signal is present. We see that the output SNR of the pro- posed adaptive beam former is greater than that of the beam former of Shan and Kailath [8].
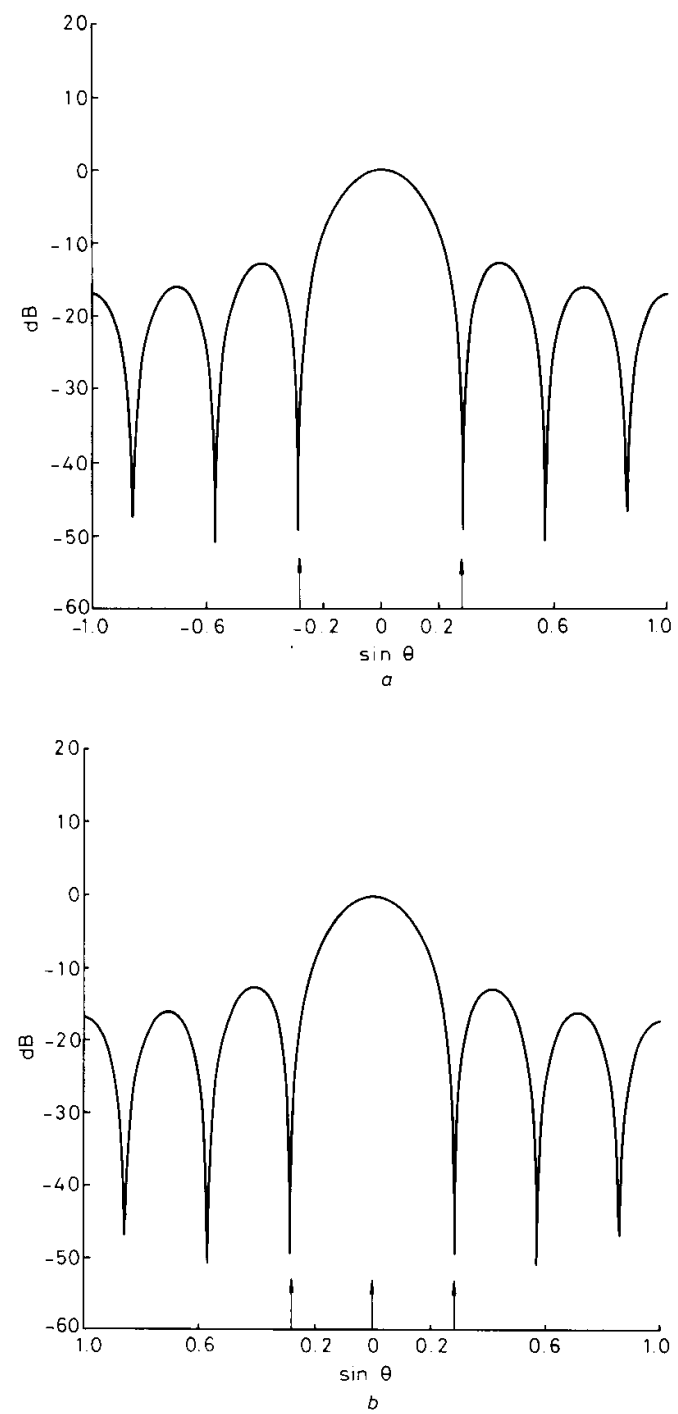

Fig. 4 Array patterns using the proposed method $a$ Desired signal absent
$b$ Desired signal present

Next, we present an example for a modulated signal to demonstrate the effectiveness of the proposed technique. In this case, the desired signal is $\rho_{s}(t) \exp \left(j \omega_{0} t\right)$ with the time-varying complex envelope $\rho_{s}(t)$ being given by $(6+j 8) \exp (-0.01 t)$. After about $2 \mathrm{~s}$, the proposed algorithm converged to its optimum. Then the resulting optimal weight was employed to operate on the current received signal data to generate the corresponding array beam pattern shown in Fig. 5. The output SNR is about $30 \mathrm{~dB}$ and the complex envelope is about $5.9+j 7.8$ for the example. It can be seen that the array pattern is almost the same as that of Fig. 4, where the desired signal is unmodulated. Therefore, the proposed technique is

IEE PROCEEDINGS, Vol. 136, Pt. F, No. 4, AUGUST 1989 
effective in the case of a modulated signal whose complex envelope is slowly time varying. All the above results confirm the theoretical work presented in Section 2.

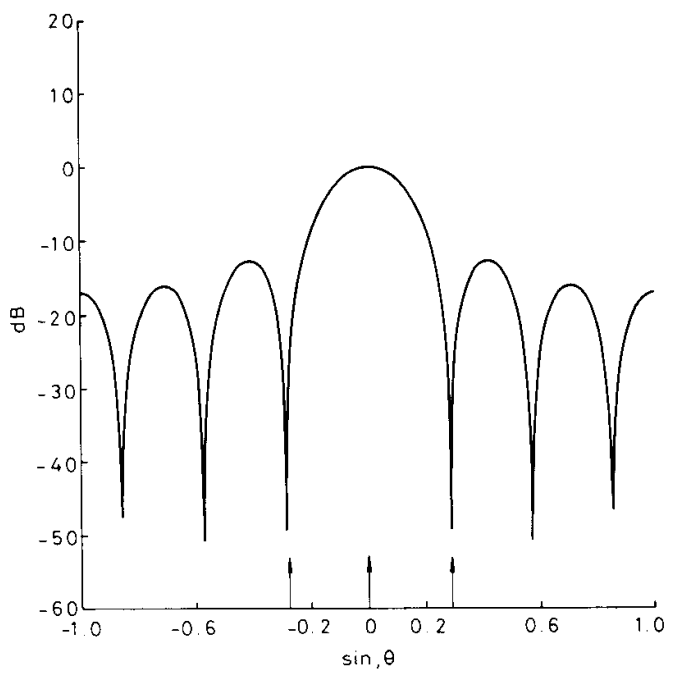

Fig. 5 Array pattern for modulated signal using the proposed method

\section{Conclusions}

In this paper, we have presented one approach to curing the signal cancellation phenomenon inherent in the spatial smoothing algorithm. The resulting adaptive beam former can not only reject coherent jammers effectively, but can also preserve the desired signal in the look direction. Theoretical analysis shows that the complex envelope of the narrowband desired signal can be found exactly as soon as the optimal constrained weight vector solution has been obtained. Moreover, the output signalto-noise ratio is also useful for determining the existence of the desired signal. Computer simulations support the theoretical work

\section{References}

1 MONZINGO, R.A. and MILLER, T.W.: 'Introduction to adaptive arrays' (Wiley, New York, 1980)

2 HOWELLS, P.W.: 'Explorations in fixed and adaptive resolution at GE and SURC', IEEE Trans., 1976, AP-24, pp. 575-584

3 APPLEBAUM, S.P.: 'Adaptive arrays', IEEE Trans., 1976 AP-24, pp. 585-598

4 WIDROW, B., MANTEY, P.E., GRIFFITHS, L.J., and GOODE B.B.: 'Adaptive antenna systems', Proc. IEEE, 1967, 55, pp. 21432159

5 APPLEBAUM, S.P., and CHAPMAN, D.J.: 'Adaptive arrays with main beam constraints', IEEE Trans., 1976, AP-24, pp. 650-662

6 FROST, O.L.: 'An algorithm for linear constrained adaptive array processing', Proc. IEEE, 1972, 60, pp. 926-935

7 GRIFFITHS, L.J., and JIM, C.W.: 'An alternative approach to linearly constrained adaptive beamforming', IEEE Trans., 1982, AP-30, pp. 27-34

8 SHAN T.-J., and KAILATH, T.: 'Adaptive beamforming for coherent signal and interference', IEEE Trans., 1985, ASSP-33, pp. $527-536$

9 EVANS, J.E., JOHNSON, J.R., and SUN, D.F.: 'Application of advanced signal processing techniques to angle of arrival estimation in ATC navigation and surveillance systems'. Lincoln Lab. Tech. Rep. 582, June 1982

10 WIDROW, B., DUVALL, K.M., GOOCH, R.P., and NEWMAN W.C.: 'Signal cancellation phenomena in adaptive antennas: causes and cures', IEEE Trans., 1982, AP-30, pp. 469-478

11 REDDY, V.U., PAULRAJ, A., and KAILATH, T.: 'Performance analysis of the optimal beamformer in the presence of correlated sources and its behaviour under spatial smoothing', IEEE Trans. 1987, ASSP-35, pp. 927-936

12 PEI, S.-C. YEH, C.-C., and CHIU, S.-C.: 'Modified spatial smoothing for coherent jammer suppression without signal cancellation', to be published in IEEE Trans., ASSP 